\title{
The Magellanic Group and the Seven Dwarfs
}

\author{
Elena D'Onghia ${ }^{\dagger}$ and George Lake \\ Institute for Theoretical Physik, University of Zurich, \\ Winterthurerstraße 190, 8057 Zurich, Switzerland \\ email:elena@physik.unizh.ch; lake@physik.unizh.ch
}

\begin{abstract}
The Magellanic Clouds were the largest members of a group of dwarf galaxies that entered the Milky Way (MW) halo at late times. This group, dominated by the LMC, contained $\sim 4 \%$ of the mass of the Milky Way prior to its accretion and tidal disruption, but $\approx 70 \%$ of the known dwarfs orbiting the MW. Our theory addresses many outstanding problems in galaxy formation associated with dwarf galaxies. First, it can explain the planar orbital configuration populated by some dSphs in the MW. Second, it provides a mechanism for lighting up a subset of dwarf galaxies to reproduce the cumulative circular velocity distribution of the satellites in the MW. Finally, our model predicts that most dwarfs will be found in association with other dwarfs. The recent discovery of Leo V (Belokurov et al. 2008), a dwarf spheroidal companion of Leo IV, and the nearby dwarf associations supports our hypothesis.
\end{abstract}

Keywords. Galaxy: halo, galaxies: clusters: general, galaxies: formation, galaxies: halos, Magellanic Clouds, cosmology: observations, dark matter

\section{Introduction}

In the cold dark matter (CDM) model, the dark halos of galaxies like the Milky Way build up hierarchically, through the accretion of less massive halos. When these subsystems avoid complete tidal disruption, they can survive in the form of satellite dwarf galaxies. However, the dwarf galaxies in the Local Group exhibit several puzzling features. Numerical simulations of CDM predict 10 to 30 times more satellites within $500 \mathrm{kpc}$ of the Milky Way and M31 than the modest observed population (e.g., Moore et al. 1999). This discrepancy between the expected and known numbers of dwarf galaxies has become known as the missing dwarf problem. The newly discovered population of ultrafaint dwarfs around the Milky Way and M31 found in the Sloan Digital Sky Survey increases by a factor of two the number of known satellites (Simon \& Geha 2007), but goes to even lower circular velocities where a comparable or even greater increase in the number of satellites is expected.

Another peculiarity is that many dwarf galaxies in the Local Group lie in the orbital plane of the Magellanic Clouds and Stream. These dwarfs have been associated with the Magellanic Clouds and termed the Magellanic Group (Lynden-Bell 1976; Fusi Pecci et al. 1995; Kroupa et al. 2005). In order to reproduce this planar configuration in the current scenario for structure formation, Libeskind et al. (2005) proposed that subhalos are anisotropically distributed in cosmological CDM simulations and that the most massive satellites tend to be aligned with filaments. Similarly, Zentner et al. (2005) suggested that the accretion of satellites along filaments in a triaxial potential leads to an anisotropic distribution of satellites.

Systems anisotropically distributed falling into the Galactic halo may not lie in a plane consistent with the orbital and spatial distribution of the MW satellites. For example, a

$\dagger$ Marie Curie fellow 
theoretical bootstrap analysis of the spatial distribution of CDM satellites (taken from a set of CDM simulations) by Metz et al. (2008) finds that even if they are aligned along filaments, they will be consistent with being drawn randomly. This could mean that alignment of the satellites along filaments may not be sufficient to reproduce the observed planar structures.

As we propose here, the origin of planar distributions is facilitated by concentrating infalling satellites into groups.

Another issue is that the dSphs of the Local Group tend to cluster tightly around the giant spirals. Proximity to a large central galaxy might prevent dwarf irregulars from accreting material, turning off star formation, and they may then undergo tidal interactions to convert them into dwarf spheroidals. However, isolated dSphs like Tucana or Cetus found in the outskirts of the Local Group (Grebel et al. 2003) suggest that dSphs might also form at great distances from giant spirals prior to their being accreted. Clues to the questions raised by these observations may be contained in measurements of the metallicities of a large sample of stars in four nearby dwarf spheroidal galaxies: Sculptor, Sextans, Fornax, and Carina. Work by Helmi et al. (2006) shows that all four lack stars with low metallicity, implying that their metallicity distribution differs significantly from that of the Galactic halo, indicating a non-local origin for these systems.

\section{Why do Magellanic Clouds need to be accreted in groups of dwarfs?}

We propose that the Magellanic Clouds and seven of the eleven dwarf galaxies around the MW were accreted as a group that was then disrupted in the halo of our Galaxy. This is supported by observations indicating that dwarfs are often found in associations and by numerical simulations where subhalos are often accreted in small groups (e.g., Li \& Helmi 2008). In particular, the LMC, SMC, and those dwarfs whose orbits are similar to those of the Magellanic Clouds may all have originally been part of such a group. This "LMC group" was dominated by the LMC and had a parent halo circular velocity of $\sim 75 \mathrm{~km} \mathrm{~s}^{-1}$ with its brightest satellite, the SMC, having a rotation velocity of $\sim 60 \mathrm{~km}$ $\mathrm{s}^{-1}$ as estimated from its $\mathrm{H}$ I distribution.

There is considerable evidence for tidal debris from the LMC group, supporting the proposal that it was tidally disrupted. The LMC and SMC have been modeled as a pair owing to their spatial proximity; as either a currently bound pair or one that became unbound on the last perigalacticon passage. The number of dwarfs assigned to the Magellanic Plane Group (Kunkel \& Demers 1976) includes the following candidates: Sagittarius, Ursa Minor, Draco, Sextans and Leo II. Of the dwarfs known before the recent flurry of discoveries, 7 out of 10 within $\sim 200 \mathrm{kpc}$ might well be part of this group. The remaining three - Fornax, Sculptor and Carina - have been proposed to be part of a second grouping (Lynden-Bell 1982).

\section{Evidence for nearby associations of dwarfs}

CDM theory predicts that many dwarf galaxies should exist in the field. Numerical simulations show that the normalized mass function of subhalos is nearly scale-free. That is, when the circular velocity distribution function of the subhalos is normalized to the parent halo, it is nearly independent of the mass of the parent. Thus, groups of dwarf galaxies are a natural expectation of CDM models on small mass scales. However, like low mass satellites, these systems are difficult to observe. 


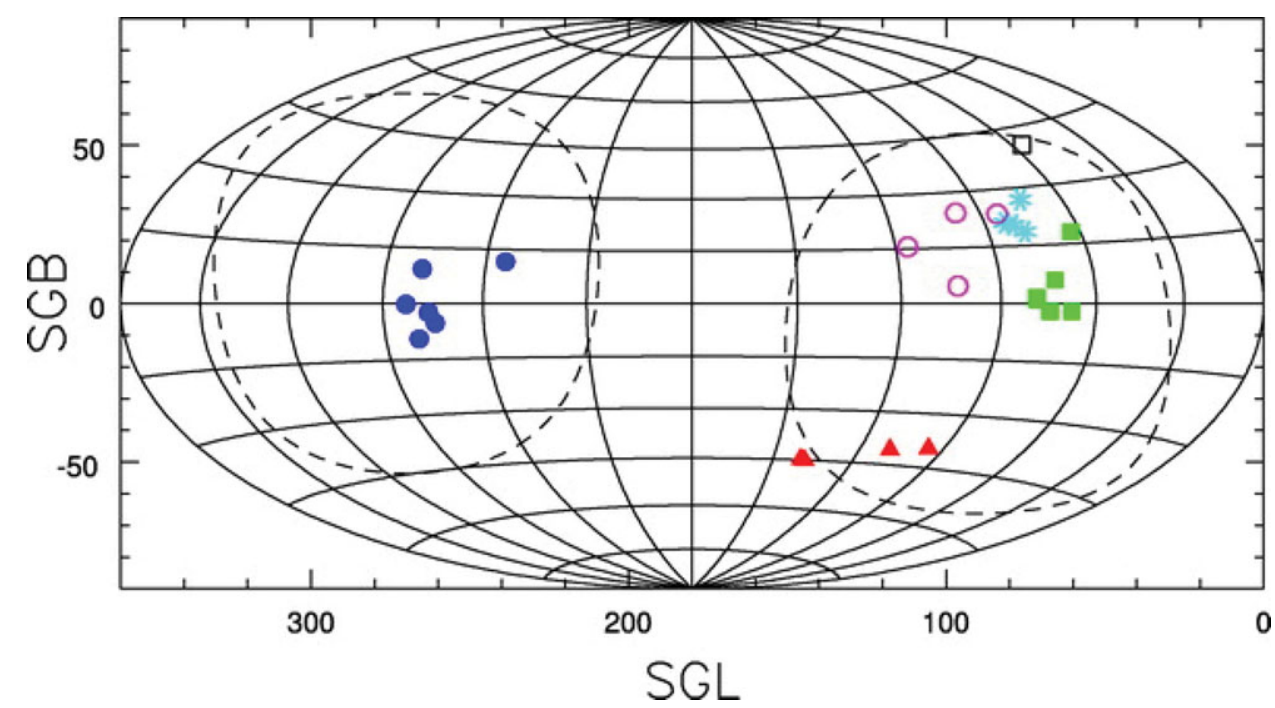

Figure 1. Distribution in supergalactic coordinates of associations of dwarfs galaxies with accurately known distances between 1.1 and $3.2 \mathrm{Mpc}$ (Tully et al. 2006).

Tully et al. (2006) discovered a number of associations of dwarf galaxies within 5 Mpc of the MW. Figure 1 displays the distribution in supergalactic coordinates of these associations with accurately known distances between 1.1 and $3.2 \mathrm{Mpc}$. These groups have properties expected for bound systems with $1-10 \times 10^{11} \mathrm{M}_{\odot}$, but are not dense enough to have virialized, and have little gas and few stars. Of the eight associations compiled by Tully et al. (2006), there are only three for which the two brightest galaxies differ by at least 1.5 magnitudes: NGC 3109, NGC 1313 and NGC 4214. In the other five, the two brightest galaxies are certain to merge if the associations collapse and virialize.

Figure 2 (left panel) shows the cumulative circular velocity distribution function inferred for the dwarf associations, the putative Magellanic Group (candidates listed previously), and the MW satellite galaxies. For each dwarf association we assume the largest dwarf galaxy circular velocity of the group to be the parent halo circular velocity. Magnitudes of member galaxies are converted to circular velocity assuming a Tully-Fisher relation in the B band (see D'Onghia \& Lake (2008) for details). The MW data includes the newest dwarfs with a minimum $\sigma=3.3 \mathrm{~km} \mathrm{~s}^{-1}$ and a correction for incomplete sky coverage (Simon \& Geha 2007).

Figure 2 shows that the nearby associations of dwarfs have a cumulative circular velocity distribution function similar to the MW, suggesting that such associations may be the progenitors of the brightest dwarf satellites in the MW. Thus, if these associations of dwarfs are accreted into larger galaxies, they can populate the bright end of the cumulative circular velocity distribution function of satellites. However, when normalized to the low mass of their parent, they have a far greater number of dwarfs.

\section{Dwarfs in the LMC group can light up more efficiently}

In our interpretation, the mass of the LMC group is $\sim 4 \%$ of the Milky Way, yet most of the dwarfs known a decade ago are associated with it. There is a similar overabundance of dwarfs in the dwarf associations. Here, we suggest that dwarf galaxies formed in LMClike groups will be luminous, while those that form by themselves in the halos of larger systems will be dark. 


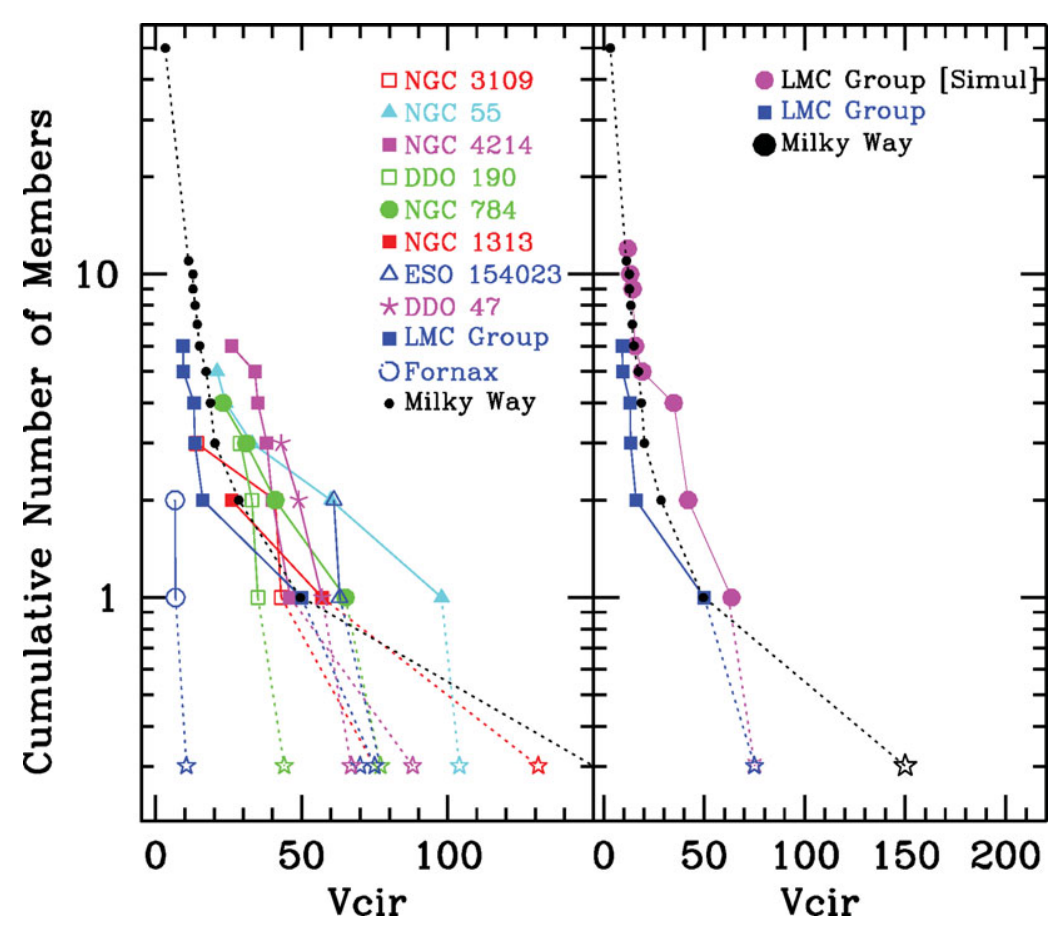

Figure 2. Cumulative circular velocity distribution of the satellites of the LMC group as compared to the nearby dwarf associations (left panel) and to the simulated LMC group in a $\Lambda$ CDM model (right panel) (see D'Onghia \& Lake 2008 for details).

It is generally assumed that galaxies with circular velocities $\sim 30 \mathrm{~km} \mathrm{~s}^{-1}$ blow out their gas. When gas is blown out of a subhalo, it eventually thermalizes to the virial temperature of the parent halo, which is $2-5 \times 10^{6} \mathrm{~K}$ for bright galaxies such as the MW. At this temperature, the cooling times are long enough that there can be a considerable reservoir of hot gas and a subhalo with a velocity scale of $10-30 \mathrm{~km} \mathrm{~s}^{-1}$ will not reaccrete much gas, and it will be dark. However, in a small parent halo like the LMC, the virial temperature is only $2 \times 10^{5} \mathrm{~K}$. This is at the peak of the cooling curve and the gas cools rapidly to $10^{4} \mathrm{~K}$. The low bulk motions in these halos might well permit reaccretion by some of the subhalos producing luminous dwarf galaxies. Note that our picture is consistent with the new proper motion measurements from Kallivayalil et al. (2006) and Kallivayalil, van der Marel \& Alcock (2006) and orbit models from Besla et al. (2007). Prior to infall, the LMC group had a virial radius of $\sim 75 \mathrm{kpc}$ and a $3-\mathrm{D}$ velocity dispersion of $\sim 100 \mathrm{~km} \mathrm{~s}^{-1}$. So, a thin plane would still be very unusual and a wide range of kinematics is expected for the disrupted satellites.

To investigate the plausibility of our model, we examined a catalog of high resolution galaxies in a cosmologically simulated volume to identify an analog to an LMC group with late infall into a MW galaxy. We note in this specific simulation that the LMC group is tidally disrupted before entering the virial radius of the MW, due to the specific mass distribution of this case. This could well be necessary to prevent the merger of the LMC and SMC prior to accretion. In Figure 2 (right panel), we display the cumulative peak circular velocity distribution of the satellites contributed by the simulated infalling group of dwarfs measured at $z=0$ within the virial radius of the MW. This is compared to the corresponding quantity for dwarfs (filled squared symbols) in the MW which may have been part of an accreted group: LMC, SMC, Sagittarius, Ursa Minor, Draco, Sextans 


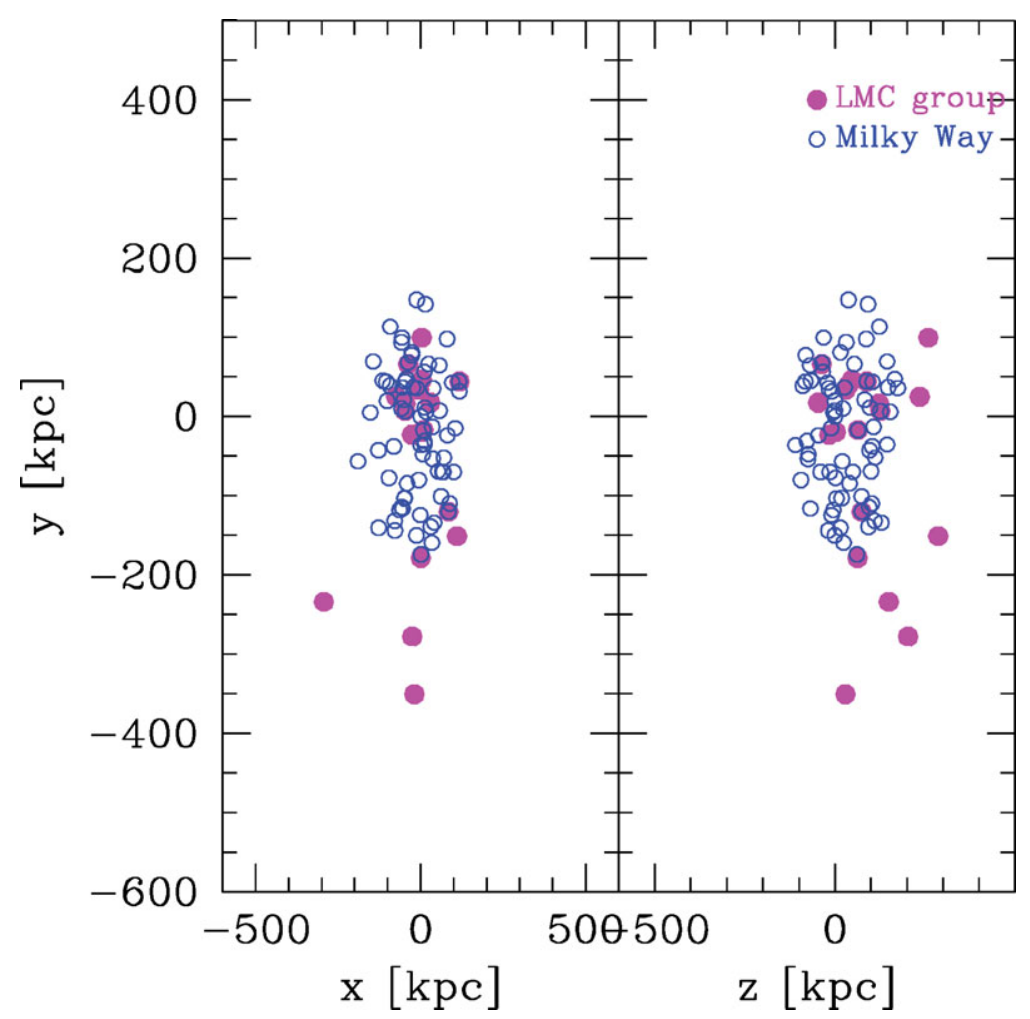

Figure 3. The spatial distribution of satellites within the virial radius of the Milky Way (blue open circles) as compared to the contributed subhalos from the break-up of the Magellanic Group at $z=0$ within $600 \mathrm{kpc}$ from the Milky Way center (magenta filled circles).

and Leo II. In Figure 2, only satellites that are accreted as part of the disrupted LMC group are displayed, because those are the dwarf galaxies that light up in our model. The remainder of the satellites that are not accreted in groups but are within the virial radius of the present-day MW are assumed to be dark.

We note that in this particular simulation, some satellites of the disrupted group are outside the MW radius at $z=0$ and some are located inside. Figure 3 shows the spatial distribution of all the satellites within the virial radius of the Milky Way (blue filled circles) as compared to the subhalos of the disrupted Magellanic group at $z=0$ (magenta stars). Despite the late infall, this particular group appears very well mixed, however almost half of the surviving subhalos of the group are at the present time located outside the virial radius of the final Milky Way. A few of them are in the outskirts of the Milky Way. These subhalos may reproduce the special cases like Tucana or Cetus that are located in low density regions of the Local Group.

\section{Conclusion}

We assume a model where the LMC was the largest member of a group of dwarf galaxies that was accreted into the MW halo. Our picture addresses several questions in galaxy formation: ( $i$ ) It explains the association of some dwarf galaxies in the Local group with the LMC-SMC system. (ii) It provides a mechanism to light up dwarf galaxies. (iii) It predicts that other isolated dwarfs will have companions. The recent discovery of Leo $\mathrm{V}$ 
(Belokurov et al. 2008), a dwarf spheroidal companion of Leo IV, and the nearby dwarf associations supports our hypothesis.

\section{Acknowledgement}

E.D. is grateful to Jacco van Loon and Joana Oliveira for organizing an interesting meeting. She also would like to thank J. Gallagher, G. Besla, K. Bekki, L. Hernquist, N. Kallivayalil, C. Mastropietro for fruitful discussions.

\section{References}

Belokurov, V., Walker, M. G., Evans, N. W., et al. 2008, ApJ, 686, L83

Besla, G., Kallivayalil, N., Hernquist, L., Robertson, B., Cox, T. J., van der Marel, R. P., \& Alcock, C. 2007, ApJ, 668, 949

D'Onghia, E. \& Lake, G. 2004, ApJ, 612, 628

D'Onghia, E. \& Lake, G. 2008, ApJ, 686, L61

Fusi Pecci, F., Bellazzini, M., Cacciari, C., \& Ferraro, F. R. 1995, AJ, 110, 1664

Grebel, E. K., Gallagher, J. S., III, \& Harbeck, D. 2003, AJ, 125, 1926

Helmi, A., Irwin, M. J., Tolstoy, E., et al. 2006, ApJ, 651, L121

Kallivayalil, N., van der Marel, R. P., Alcock, C., Axelrod, T., Cook, K. H., Drake, A. J., \& Geha, M. 2006, ApJ, 638, 772

Kallivayalil, N., van der Marel, R. P., \& Alcock, C. 2006, ApJ, 652, 1213

Kroupa, P., Theis, C., \& Boily, C. M. 2005, A\&A, 431, 517

Kunkel, W. E. \& Demers, S. 1976, RGOB, 241

Li, Y. \& Helmi, A. 2008, MNRAS, 385, 1365

Libeskind, N. I., Frenk, C. S., Cole, S., Helly, J. C., Jenkins, A., Navarro, J. F., \& Power, C. 2005, MNRAS, 363, 146

Lin, D. N. C. \& Lynden-Bell, D. 1982, MNRAS, 198, 707

Lynden-Bell, D. 1976, MNRAS, 174, 695

Lynden-Bell, D. 1982, Obs, 102, 7L

Metz, M., Kroupa, P., \& Libeskind, N. I. 2008, ApJ, 680, 287

Moore, B., Ghigna, S., Governato, F., Lake, G., Quinn, T., Stadel, J., \& Tozzi, P. 1999, ApJ, 524,19

Piatek, S., Pryor, C., Bristow, P., Olszewski, E. W., Harris, H. C., Mateo, M., Minniti, D., \& Tinney, C. G. 2005, $A J, 130,95$

Simon, J. D. \& Geha, M. 2007, ApJ, 670, 313

Tully, R. B., Rizzi, L., Dolphin, A. E., et al. 2006, AJ, 132, 729

Zentner, A. R., Kravtsov, A. V., Gnedin, O. Y., \& Klypin, A. A. 2005, ApJ, 629, 219 